\title{
Telephone or surgery asthma reviews? Preferences of participants in a primary care randomised controlled trial
}

\author{
Hilary Pinnock ${ }^{\mathrm{a}, *}$, Victoria Madden ${ }^{\mathrm{b}}$, Caroline Snellgrove ${ }^{\mathrm{c}}$, \\ Aziz Sheikha
}

a Division of Community Health Sciences: GP section, University of Edinburgh, 20, West Richmond St, Edinburgh, EH8 9DX, UK

b Grimshill, 1 Borstal Hill, Whitstable, Kent, UK

c Whitstable Medical Practice, Harbour Street, Whitstable, Keit C

Received 21 June 2004; accepted 31, Octoj) (2)

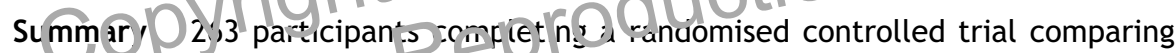
telephone vs. face-to-face astimi consultations were asked about preferences for future reviews. Qualitative analysis of data from 209 respondents identified divergent views. Clear opinions were expressed about the respective roles of the two modes of consulting; telephone consultations were considered convenient for reviewing 'well controlled' asthma, whereas face-to-face consultations were perceived as allowing in-depth assessment of problems in those with more symptomatic asthma. Practices may consider offering patients the choice of a telephone or face-to-face review.

(c) 2004 General Practice Airways Group. Published by Elsevier Ltd. All rights reserved.

\section{Introduction}

The evidence-based recommendation of national and international guidelines, that people with asthma should be reviewed regularly, reflects good clinical practice $[1,2]$. Only about a third of

* Corresponding author. Present address: Whitstable Health Centre, Harbour Street, Whitstable, Kent CT5 1BZ, UK.

Tel.: +44 1227 594400; fax: +44 1227771474 .

E-mail address: hpinnock@gpiag-asthma.org (H. Pinnock). people with asthma heed this advice and attend their general practice for a routine annual review $[3,4]$. Our trial of telephone consultations for asthma demonstrated that telephone-based care can facilitate delivery of routine healthcare, substantially increasing the proportion of patients reviewed when compared with traditional faceto-face consultations [5]. We aimed to explore the preferences expressed by people with asthma for alternative modes of consultation in order to gain insight into factors which may influence the 
implementation of services offering a telephone option.

\section{Methods}

\section{Setting}

Our randomised controlled trial comparing telephone and face-to-face consultations for delivering routine asthma care, undertaken in four UK general practices experienced in the provision of proactive asthma care, recruited 278 symptomatic asthma patients [mean age: 55.5 yrs (SD 17.5), 58\% female] [5]. During the trial, 101 of the patients experienced a telephone asthma consultation and 68 had a face-to-face review with the practice asthma nurse. Fifteen patients withdrew during the course of the 3-month study.

\section{Study procedure}

On completion of the trial, we posted a semistructured questionnaire to the 263 participants who completed the study, enquiring about their preferences for future reviews, and the reasons for their preference. Non-responders were ser $t$ two reminders. Approval was ob ain ron the SouthEast Multi-centre Research Etnics Committee. C C

\section{Analysis}

Patients' free-text responses to open-ended questions about their views on modes of consultation were thematically analysed through a multidisciplinary discussion involving a general practitioner, an asthma nurse, an asthma patient and a health services researcher. Using the principles of qualitative content analysis, we developed a coding frame and identified key emerging themes [6]. These themes were presented to attendees at a feedback meeting for patients of one of the practices, and areas of agreement or disagreement with our preliminary themes were identified through open discussion. This discussion was simultaneously transcribed and data analysed for convergent and divergent themes.

\section{Results}

We obtained responses to our questionnaire from 209/263 (79\%) of those canvassed (mean age: 54.9 years (SD 17.5); female: $n=153(60 \%)$ ). The subsequent feedback meeting was attended by two trial participants, 10 non-study patients, the practice asthma nurse and the practice manager of one of the participating practices.

Overall 70/209 (33\%) preferred telephone consultations for future reviews, 35/209 (17\%) preferred surgery and 104 (50\%) expressed no preference. Preferences for riode of ff ture consultation analvsed $\mathrm{bx} /$ Gge, gender, trial allocation and lievious experience of telephone rons 4 taton are provided $n$ Table 1.

Five mairl the nes viere Identified from the free text essonses.

\section{Convenience of telephone consultations}

Telephone consultations were valued by many of the respondents as being convenient for those at work or with domestic commitments, helping to overcome mobility and transport problems and reducing time and travel costs.

"Due to working full time, difficult to get an appointment when convenient to me" (38 M. Trial

Table 1 Preferences for mode of future consultation analysed by age, gender, allocation and experience of telephone consultation.

\begin{tabular}{lllll}
\hline & & Preference & & \\
\cline { 2 - 4 } & & Surgery & Telephone & No preference \\
\hline All & $(n=209)$ & $35(17 \%)$ & $70(33 \%)$ & $104(50 \%)$ \\
Allocation & Surgery $(n=103)$ & $18(17.5 \%)$ & $27(26.2 \%)$ & $58(56.3 \%)$ \\
Consultation experience & Telephone $(n=106)$ & $17(16.0 \%)$ & $43(40.6 \%)$ & $46(43.4 \%)$ \\
& Surgery $(n=58)$ & $16(27.6 \%)$ & $11(19.0 \%)$ & $31(53.4 \%)$ \\
Gender & Telephone $(n=85)$ & $10(11.8 \%)$ & $37(43.5 \%)$ & $38(44.7 \%)$ \\
Age & Female $(n=116)$ & $17(14.7 \%)$ & $42(36.2 \%)$ & $57(49.1 \%)$ \\
\hline & Male $(n=93)$ & $18(19.4 \%)$ & $28(30.1 \%)$ & $47(50.5 \%)$ \\
& Mean $(S D)$ & $59.5(16.0)$ & $53.0(18.6)$ & $58.6(15.9)$ \\
\hline
\end{tabular}


allocation: telephone review. Future preference: telephone)

"Because I have a sick husband and I don't leave him more than I can help" (76 F. Trial allocation: telephone review. Future preference: telephone)

"Totally dependent on others for transport; find public transport too unreliable" $(88 \mathrm{M}$. Trial allocation: telephone review. Future preference: telephone)

Several responses suggested that the convenience of telephone consultations may facilitate a review that would otherwise have been postponed, sometimes to the detriment of care.

"As one tends to put off going to the surgery as one "isn't bad enough", a regular telephone check would be ideal" (78 F. Trial allocation: telephone review. Future preference: telephone)

"As my asthma is not severe I found the phone consultation very convenient. I perhaps would not have gone to the surgery otherwise and would still be suffering now" (43 F. Trial allocation: telephone review. Future preference: telephone)

\section{Specific probtiens writh telephone} cons(1): \pm ions

Concerns were occasionally expressed about confidentiality, particularly when calls were taken at work, or the timing was inconvenient.

"I work full time and it's difficult to talk privately about a medical condition because people are often in the same office/location" (42 F. Trial allocation: telephone, but review not achieved. Future preference: surgery)

"I was not prepared for the phone call and should have made a note of what was said" (79 M. Trial allocation: telephone review. Future preference: telephone)

\section{Human dimension of face-to-face encounters}

About half of those preferring surgery consultations appreciated the personal, relaxed style, which was perceived as being more 'human'. Some respondents commented on the importance of visual clues contributing to a more in-depth check.
"I prefer talking to the nurse than on the phone" (32 F. Trial allocation: telephone review. Future preference: surgery)

"Being on the phone puts you on the spot. It's nice to discuss problems face to face in relaxed environment" (38 M. Trial allocation: surgery review. Future preference: surgery)

'Direct contact with experts' eye is always more helpful" $(85 \mathrm{M}$. Trial allocation: telephone, but review not achieved. Future preference: surgery)

\section{Appropriate mode of consultation depended on the clinical situation}

Many respondents felt that, as their asthma was mild and well controlled, quick and convenient telephone reviews were ideal. If a problem arose with their asthma they would make an appointment at the surgery.

[The telephone is] convenier t. if Qdul Pave a problem with your cstina you make an appointment a the cidctors" (29 F. Trial allocation: teleDhone review. Fut re preference: telephone)

"A routine telephone check would be reassuring \& any questions could be asked. But for specific new symptoms the face-to face meeting is preferred" (78 F. Trial allocation: telephone review. Future preference: telephone)

\section{Wider implications}

A few patients wanted to explore other innovative modes of consultation.

"I have attached peak flow information. Would it be possible to e-mail?" ( $49 \mathrm{M}$. Trial allocation: surgery review. Future preference: surgery)

Patients at the feedback meeting agreed with our conclusions, but in addition expressed interest in extending telephone care to other chronic diseases.

"Good to have an option that might be more convenient" (non-study participant at the feedback meeting)

"Potential for wider applicability and greater access to consultation" (non-study participant at the feedback meeting) 
"General applicability of telephone consultationnot just asthma" (study participant at the feedback meeting)

\section{Discussion}

Our results suggest that participants perceived complementary roles for telephone and surgery reviews. Telephone consultations were seen as a convenient option for the routine review of 'mild' or 'well-controlled' asthma, overcoming work and domestic obstacles and facilitating acceptance of care. The personal nature of face-to-face consultations, allowing in-depth assessment, was seen as being more appropriate if asthma control had deteriorated.

\section{Limitations and strengths of our study}

The responses to a single free-text question at the end of a trial can only provide limited insight; however, the comments do indicate the potential value of a follow-on in-depth exploration of patients' perspectives on the mode of delivery of their care. Despite the broad entry criteria for our trial, our volunteer sample were slightt' olcer than the total eligible posutátin [7 and may have been favouratill aisposed to the concep of tele oho ie con:uitations and thereiciont wholly representative of the wider population. However, the feedback group, which included non-study patients, endorsed wider applicability.

Strengths of our study include the large sample size, and the fact that many of the participants had previous experience of telephone consultations which informed their opinions.

\section{Interpretation and practical implications}

Our participants' views echoed recognised features of telephone communication [7]. Patients who preferred 'personal' face-to-face consultations may have been uncomfortable with the 'distance' imposed by a telephone conversation. The preference of some patients for the 'relaxed', 'in depth' style of face-to-face consultations may reflect the more focused nature of telephone conversations. This was not necessarily a problem for a routine check-up of 'controlled' asthma, but may explain the preference for a surgery appointment if asthma had deteriorated. A greater understanding of the dynamics of telephone consultations would facilitate specific training to help professionals overcome some of these issues [8].
Several practical implementation issues were highlighted. Some patients felt unprepared for the call, and others were concerned about confidentiality at their workplace. Our trial procedure expected the nurses to initiate the calls; these problems could be overcome if patients were able to book telephone consultations at their convenience.

\section{Conclusions}

This preliminary enquiry suggests that many of our patients were positive about the advantages of telephone consultations and expressed clear opinions about the respective roles of telephone and surgery asthma reviews. Pending further exploration through an in-depth qualitative interview study, practices may consider offering patients the choice of a face-to-face or telephone review.

\section{Funding}

British Lund Fild indution (Grant No P00/9). AS vas ilfporied by aNHS/Pi)P National Primary Care Award.

\section{conflicts of interest}

None known.

\section{Acknowledgments}

This study was originally developed at a research meeting of the General Practice Airways Group, which was organised by Dr Mark Levy and funded by an educational grant from AstraZeneca. Jane Scullion undertook quality control visits to the practices. Professor David Price advised on the project. The study was undertaken by: Dr Robert Bawden and Frances Yates, Botesdale Health Centre, Diss, Norfolk; Dr Stephen Proctor and Carol Howard, Clarendon Medical Centre, Hyde, Cheshire; Stephanie Wolfe, Thorpewood Surgery, Norwich, Norfolk; and Caroline Snellgrove, Whitstable Medical Practice, Kent.

\section{References}

[1] The British Thoracic Society/Scottish Intercollegiate Guideline Network. British guideline on the management of asthma. Thorax 2003;58(S1):i1-94. 
[2] Global Strategy for Asthma Management and Prevention, GINA Workshop Report: updated November 2003. Available on http://ginasthma.com/.

[3] Gruffydd-Jones K, Nicholson I, Best L, Connell E. Why don't patients attend the asthma clinic? Asthma Gen Pract 1999;7:36-8.

[4] National Asthma Campaign. Out in the open: a true picture of asthma in the United Kingdom today. Asthma J 2001;6:3-14.

[5] Pinnock H, Bawden R, Proctor S, Wolfe S, Scullion J, Price D, et al. Accessibility, acceptability and effectiveness of tele- phone reviews for asthma in primary care: randomised controlled trial. BMJ 2003;326:477-9.

[6] Bryman A. Qualitative data analysis. In: Social Research Methods. Oxford University Press; 2001, pp 381403.

[7] Rutter DR. Communicating by telephone. Oxford: Pergamon Press; 1987.

[8] Car J, Sheikh A. Telephone consultations. BMJ 2003;326: 966-99.

Available online at www.sciencedirect.com

science $\boldsymbol{d}$ Direct.

\section{Available online at http://www.thepcrj.com}

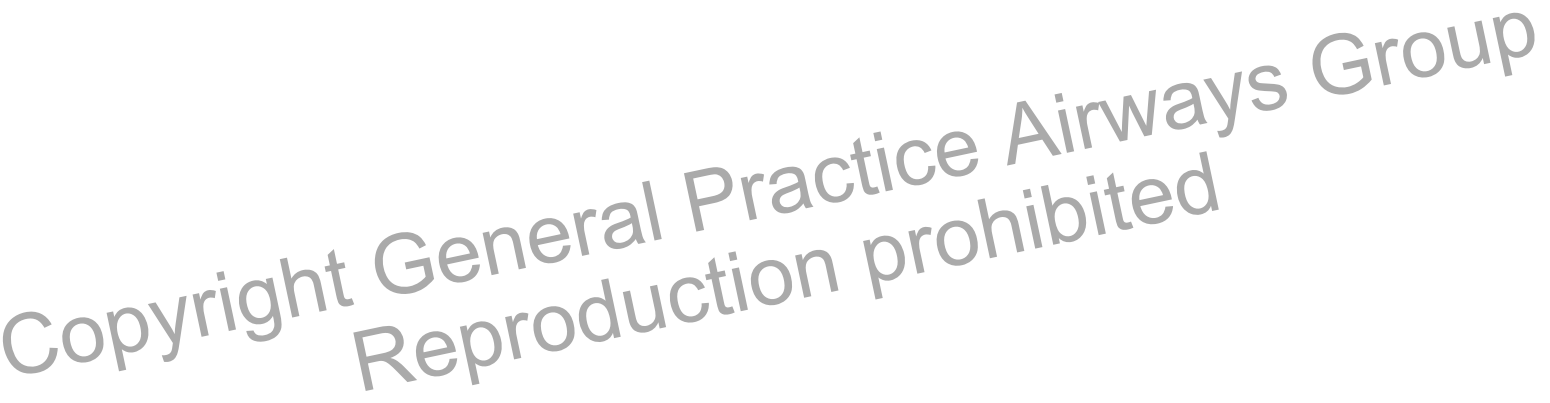

